\title{
Attending to color and shape: The special role of location in selective visual processing
}

\author{
YEHOSHUA TSAL and NILLI LAVIE \\ Tel Aviv University, Tel Aviv, Israel
}

\begin{abstract}
Subjects were presented with circular arrays of letters and were instructed to report first a given target (or targets) and then any other letters they could identify. The target(s) was (were) a letter of a given color (Experiment 1) or a given shape (Experiment 2), or two letters of a given shape (Experiment 3). In all three experiments, the additional letters reported tended to be adjacent to the first reported target(s). The results suggest that the selective processing of targets specified by color or by shape is accomplished by attending to the targets' locations.
\end{abstract}

The role of stimulus location in the selective processing of visual information has attracted much interest in recent years (e.g., Duncan, 1981, 1984; Eriksen \& Hoffman, 1973; Hoffman \& Nelson, 1981; Kahneman \& Henik, 1981; Nissen, 1985; Posner, Snyder, \& Davidson, 1980; Sagi \& Julesz, 1985; Treisman \& Gelade, 1980; Tsal, 1983) and has evolved into an implicit controversy. Some researchers believe that spatial location plays a unique role in the selection of information for further processing (e.g., Posner et al., 1980), whereas others claim that it is just one selection dimension (although an extremely efficient one) that is not different in principle from other stimulus dimensions, such as color or shape (e.g., Duncan, 1981).

Theories of attention do not generally ascribe a special role to location. It has generally been assumed that selective processing is accomplished either by admitting relevant information into one particular channel, defined by any given physical attribute (Broadbent, 1958), or by activating internal structures responding selectively to a given attribute (e.g., LaBerge, 1975). Stimulus location is not uniquely entailed in the process unless it defines the relevant selection attribute.

Early studies in the visual modality using partial report (e.g., von Wright, 1970) and visual search (e.g., Estes, 1972) generally supported this view by showing that selective processing could be carried out with minimal interference from nontarget elements as long as the target(s) was (were) clearly distinguishable on any given physical dimension (e.g., location, color, shape, size).

However, more recent studies (e.g., Eriksen \& Hoffman, 1974; Posner et al., 1980) suggested a special role for spatial location, as they found that advance knowledge of target location improved the processing of that target, even in the absence of any distractors in the field. These findings suggest that attention operates as a "spotlight"

We are grateful to Robert Lubow for his helpful comments on an earlier draft. Requests for reprints should be addressed to Yehoshua Tsal, Department of Psychology, Tel Aviv University, Ramat Aviv 69 978, Tel Aviv, Israel. that "illuminates" a given small area within which stimuli are processed in detail (e.g., Eriksen \& Hoffman, 1973). On the other hand, the comparative efficiency of advance knowledge of other stimulus properties has been somewhat controversial (e.g., Duncan, 1984). Posner et al. (1980) observed no facilitation in performance when subjects were told in advance which letter would be presented (i.e., form preknowledge). Barber and Folkard (1972) found that reducing the size of a letter set from which a target could be selected improved performance, and Humphreys (1981a) reported that even when stimulus location was known in advance, color preknowledge could further facilitate performance.

Strong support for the spotlight notion was provided by Hoffman and his associates (Hoffman \& Nelson, 1981; Hoffman, Nelson, \& Houck, 1983). Their subjects searched four-letter displays for prespecified targets. They were also instructed to locate a missing side of a box that appeared near one of the letters. Performance on the search task was more accurate when the box was adjacent to the target than when it was not. The authors reasonably concluded that the box attracted attention to its vicinity, thereby improving performance for adjacent targets. However, it has been suggested elsewhere (Duncan, 1984) that this finding reflects the strength of perceptual grouping rather than adjacency per se.

Further evidence for the special role of location comes from studies that show that the locational arrangement of items may be important even when relevant and irrelevant items are distinguished on the basis of another property (Fryklund, 1975; Kahneman \& Henik, 1977, 1981). For example, Kahneman and Henik (1981) presented two rows containing blue and red letters in various arrangements and instructed subjects to ignore the red letters and report the blue ones. Selective performance was best when the blue letters formed a coherent group (e.g., a single row). In a related earlier study, Lappin (1967) found more accurate performance for reporting three attributes of a single object, than for reporting the same attributes individually in three spatially separated objects. Although these findings emphasize the special role 
of stimulus location, they do not provide direct support for the spotlight theory of attention, as they suggest that attention may be allocated to perceptually organized groups, and not necessarily to adjacent items.

Recently, Treisman and Gelade (1980) and Nissen (1985) suggested a specific role for stimulus localization. These investigators proposed that individual features may register preattentively without their location having been perceived, but the integration of features from different dimensions (e.g., color and shape) into unified objects is mediated by their localization. However, the evidence for this proposition is not strong. Treisman and Gelade (1980) observed that identification of a target distinguished from distractors on the basis of a single feature was well above chance even when the target was mislocated. In contrast, mislocated targets specified by the conjunction of two features (e.g., a green $T$ in a background of green $\mathrm{Xs}$ and brown Ts) were not identified at levels above chance. However, as Nissen (1985) pointed out, these results can be alternatively explained by the fact that identifying conjunctive targets was simply more difficult, resulting in the reduction of correct identifications with incorrect localizations (Baron, 1973).

Nissen (1985) presented four items varying in location, color, and shape. In one condition, the subjects were to report the color and shape of items cued by location. Responses for color and shape were found to be independent. In another condition, the subjects were to report the shape and location of items cued by their color. In this condition, localization of the cued items was required for correct selection of the corresponding shapes. Evidently, localization of items mediated the integration of the selected feature (color) and the reported feature (shape). It should be noted, however, that these results do not preclude the possibility that localization is also required for identification of single features. Nissen could have tested this possibility with a relevant condition in which subjects were presented with a given shape (color) following the display, and were asked to determine whether this shape (color) appeared in the display and, if so, where it was located. It would be interesting to know whether identification of a single feature is contingent upon its localization.

In an earlier study, Snyder (1972) tested a proposition of von Wright (1970) that ascribed a more general role to stimulus localization. These investigators postulated a two-stage model of selective processing in which the localization of selected targets is required for their identification. Snyder presented a circular array of letters and instructed subjects to report the shape and location of a target specified by color, fragmentation, or inversion. The error data revealed that when subjects falsely reported a nontarget item it was more often from a position adjacent to the target than from a remote one. Snyder concluded that the fact that attention was directed at the location of the target indicates that targets specified by a physical cue need to be localized in order to be identified. This conclusion is unwarranted. First, Snyder's subjects were required to localize, as well as identify, the target. Thus, directing attention to location need not have mediated target identification but may simply have been required for the localization response itself. Second, as observed in Nissen's (1985) experiment, attention to location may have been required for integration of the cued feature (e.g., color) with the reported feature (letter shape). Third, conclusions based exclusively on error data may be misleading in this context. Subjects may be flexible in attending to various stimulus dimensions (e.g., Duncan, 1984); therefore, Snyder's subjects might have attended to different properties on different trials. Since error responses reflected unsuccessful performance, it is not certain that attending to location mediated successful selective processing.

In the present study we explored the possibility that attending to location is a general and mandatory process that is not restricted to tasks that precue the locus of a stimulus. Specifically, our purpose was to investigate whether attention is allocated to location even when this dimension is irrelevant to the task, that is, when the target is prespecified by color or by shape. The task we used combined visual search followed by free report. We presented circular arrays of letters and instructed the subjects to report first a letter of a given color or a given shape, and then any other letters they could identify. The free report of letters was intended to reveal which attribute of the first reported target was attended to. For example, if subjects attended to the shape that specified the target, then letters in the display that were similar in shape to the target should have been more likely to be reported than other, nonsimilar, letters.

\section{EXPERIMENT 1}

In the first experiment we presented a circular array containing a mixture of three red, three green, and three brown letters. The subjects were instructed to report first a single letter of a given color, and then any other letters they could identify. If attention is allocated to the location of the first letter, then letters adjacent to it should be reported more frequently than neutral letters in the display. Similarly, if attention is allocated to the color of the first reported letter, then letters of the same color should be reported more frequently than the neutral letters.

\section{Method}

Subjects. The subjects were 8 undergraduates fulfilling a course requirement. All had normal or corrected-to-normal vision.

Stimuli. The stimuli were presented in a two-field Gerbrands tachistoscope. There were 36 circular arrays of nine uppercase letters randomly sampled from the entire English alphabet, with the constraint that no letter be repeated in a display. In each array three letters were red, three were green, and three were brown. The colors were randomly paired with the various letters, with the constraint that letters of the same color never be adjacent.

Each letter subtended $1^{\circ}$ in height and $0.7^{\circ}$ in width of visual angle, and the contour-to-contour interletter distance was $1.2^{\circ}$ of visual angle. The entire array subtended $6.6^{\circ}$ of visual angle in diameter.

Procedure. Each stimulus was presented for $100 \mathrm{msec}$, and was immediately preceded by a central fixation cross presented for $1 \mathrm{sec}$. 
The subjects were instructed to report first a single letter of a given color (e.g., "Report first any one of the three red letters"), and then any other letters they could identify. It was emphasized that the purpose of the secondary task was to report as many of the letters in the display as possible; therefore, the letters might, but need not, be the same color as the first reported letter. The subjects were given three blocks, each containing the same 36 displays. In one block the letter to be reported first was red, in another block it was green, and in another it was brown. The order of block presentation was randomized across subjects. Each block was preceded by a few practice trials.

\section{Results and Discussion}

A preliminary inspection of the data indicated no differences in performance for the three different colors. Thus, the data for each subject were collapsed over the three blocks of trials. Those trials in which the first reported letter was not of the instructed color (approximately 16\% on the average) were excluded from the analysis. Table 1 shows the mean number of correct letters per trial reported in addition to the first letter for each category, as well as the mean number of errors per trial. The location category includes letters from the two positions adjacent to the first reported letter. The color category includes the two letters of the same color as the first letter. The neutral category includes letters from the remaining positions of the display. There were two possible location letters, two possible color letters, and four possible neutral letters. To adjust for unequal chance responses, the mean numbers of reported location letters and color letters were multiplied by 2 for each subject. These corrected numbers were used in the analyses, which indicated that location letters were reported more frequently than neutral letters $[t(7)=3.45, p<.01]$, but that the difference between color letters and neutral letters reported was not significant $[t(7)=1.02]$. The difference between location letters and color letters reported was also significant $[t(7)=3.26, p<.01]$, but this is of less concern here. These findings suggest that attention is allocated to the location of the stimulus even when the location dimension is irrelevant to the task. A further analysis was performed within the neutral category. No difference in the proportion of reported letters was found between the two neutral positions closer to the target and the two that were more distant from it $[t(7)=1.22]$. Thus, there was no evidence for a spatial distance effect beyond that observed for the two adjacent location letters.

A potential problem for the present interpretation is that maintaining fixation at the center of the display may not have corresponded to the optimal strategy for performing the task. Since three of the letters were always rele-

Table 1

Experiment 1: Mean Number of Letters per Trial Reported in Each Category in Addition to Target

\begin{tabular}{cccc}
\multicolumn{3}{c}{ in Each Category in Addition to Target } & \\
\hline Location & Color & Neutral & Error \\
\hline 1.28 & 0.58 & 0.50 & 0.23 \\
$0.64^{*}$ & $0.29^{*}$ & &
\end{tabular}

*Uncorrected means. vant, the subjects may have shifted fixation to any array position prior to stimulus onset, since any random position was highly likely to include a letter of the relevant color in that or in an adjacent position. If subjects indeed shifted their gaze to one of the letter positions, then the superiority of location letters may reflect not the effect of attention, but the fact that these letters were simply closer to the fovea than the remaining letters. In addition to extending the basic finding of Experiment 1, in Experiments 2 and 3 we also attempted to eliminate the above alternative interpretation.

\section{EXPERIMENT 2}

In Experiment 2, the subjects were presented with displays containing only brown letters. Two of the letters were "curved" (selected from the following set of letters: $\mathrm{D}, \mathrm{G}, \mathrm{O}$, and $\mathrm{Q}$ ). The subjects were instructed to report first one of the curved letters, and then any other letter they could identify.

This experiment had three goals: The first was to extend the findings of Experiment 1 to the shape dimension. The second was to investigate whether attending to stimulus location is limited to tasks that require the conjunction of features from different dimensions (e.g., Nissen, 1985). One might argue that because, in Experiment 1, the subjects had to select a letter by color but report its shape, the task required the conjunction of color and shape. In Experiment 2, both the selection and the response criteria were confined to the shape dimension. Thus, if subjects reported letters adjacent to the first letter, this would indicate that attending to location is involved in the perception of individual features, and not only in their conjunction. The third purpose of the experiment was to minimize the likelihood of subjects' shifting their fixation from the center of the display. Since there were only two relevant letters in a display, the probability of a relevant letter appearing in a randomly selected position or a position adjacent to it was largely reduced.

\section{Method}

Subjects. The subjects were 10 undergraduates fulfilling a course requirement. All had normal or corrected-to-normal vision.

Stimuli. The stimuli were presented on 36 cards, 18 of which were presented twice to produce a total of 54 trials. Each card contained a circular array of nine brown letters. Each array contained two curved letters randomly selected from the following set: D, G, $O$, and $Q$. The two curved letters were randomly assigned to the various positions, with the constraints that they never be adjacent and that they never be separated by more than two intervening letters. The latter constraint was added to further reduce the chance that any given random position or a position adjacent to it would contain a curved letter. To maximize the physical salience of the two curved letters, the remaining letters in the display contained no curved features. These angular letters were randomly selected from the following set: A, E, F, H, I, K, L, M, N, T, V, W, X, $Y, Z$. The visual angles subtended by the letters, the interletter spaces, and the entire array were identical to those of Experiment 1.

Procedure. The subjects were given a single block containing all 54 trials. They were instructed to report first either of the two 
curved letters and then any other letters they could identify. It was emphasized that the purpose of the secondary task was to report as many of the letters as possible; therefore the letters might, but need not, include the second curved letter. In all other respects, the procedure was identical to that of Experiment 1.

\section{Results and Discussion}

In spite of explicit instructions to report a curved letter first, all subjects reported an angular letter first on some of the trials. For some subjects these trials reached a substantial proportion (about one-third) of the trials. It is possible that the first reported angular letter was processed while the subject was searching for a curved letter. Alternatively, it is possible that a curved letter was always identified first, but that the order of report did not correspond to the order of processing. A preliminary inspection indicated the same pattern of results for trials in which a curved letter was reported first and for those in which an angular letter was reported first. Thus, both types of trials were included in the analyses. For those few trials in which an angular letter was reported first and the two curved letters were reported later, the second reported curved letter was recorded as a shape response. Those occasional trials in which an incorrect curved letter was reported first, or in which only angular letters were reported, were excluded from the analyses. Table 2 shows the mean number of correct letters reported per trial in addition to the first letter for each category, as well as the mean number of errors per trial for the curved and angular letters. The location category includes letters reported from positions adjacent to the first reported curved letter, the shape category includes the second reported curved letters, and the neutral category includes the remaining letters. For each display there were two possible location letters, one possible shape letter, and five possible neutral letters. To adjust for unequal chance responses, for each subject the number of reported location letters was multiplied by 2.5 , and the number of reported shape letters was multiplied by 5 , prior to the analyses.

The analyses indicated that location letters were reported more frequently than neutral letters $[t(9)=3.68$, $p<.005]$ and that the difference between shape letters and neutral letters reported was not significant $[t(9)=$ 0.95 ]. The difference between location letters and shape letters reported did not reach significance $[t(9)=1.66]$. The findings are quite similar to those of Experiment 1. They clearly indicate that when subjects are instructed to report a letter of a given shape, attention is allocated primarily to the letter's location. Hence, the present ex-

Table 2

Experiment 2: Mean Number of Letters per Trial Reported in Each Category in Addition to Target

\begin{tabular}{ccccc}
\hline & & & \multicolumn{2}{c}{ Error } \\
\cline { 4 - 5 } Location & Shape & Neutral & Angular & Curved \\
\hline 1.59 & 0.96 & 0.78 & 0.17 & 0.03 \\
$0.64^{*}$ & $0.19^{*}$ & & & \\
\hline
\end{tabular}

*Uncorrected means. periment extended the findings of Experiment 1 to the shape dimension. More importantly, since in the present experiment both the selection and the response criteria were restricted to the shape dimension, the results indicate that attending to location not only mediates the conjunction of features from different dimensions, but is involved in processing features from a single dimension as well. As in Experiment 1, there was no difference in the proportion of reported letters between the two neutral letters closest to the target and the two that were most distant from it $[t(7)=0.88]$.

\section{EXPERIMENT 3}

In Experiment 3 we presented displays similar to those of Experiment 2, but instructed subjects to report the two curved letters first, and then any other letters they could identify. Furthermore, exposure duration was reduced to $50 \mathrm{msec}$. We hoped that severely limiting exposure duration would produce a sufficient number of trials in which only one curved letter could be identified. The purpose of this experiment was to bias subjects as much as possible toward directing attention to the shape (roundness) of the target letters and against directing attention to their locations. First, attempting to perceive the two curved letters under constrained presentation conditions should maximize the operation of selective attentional mechanisms responding to shape. Second, in this experiment, unlike Experiments 1 and 2, focusing attention on the location of a single array position would be detrimental to the task, since subjects were instructed to report the two curved letters, which could occupy relatively remote locations. If location superiority were obtained under these conditions it would strengthen the proposed interpretation of the present study.

\section{Method}

Subjects. The 10 subjects who had participated in Experiment 2 were used in this experiment.

Stimuli. The stimuli were the same as those of Experiment 2. Procedure. The procedure was identical to that of Experiment 2, with two exceptions. First, exposure duration was $50 \mathrm{msec}$. (For 1 subject, who had difficulty in identifying letters from the displays, the duration was increased to $70 \mathrm{msec}$.) Second, the subjects were instructed to report first the two curved letters and then any other letters they could identify. They were further told that if they could not identify curved letters, they should still report any other letters they saw.

\section{Results and Discussion}

Again, some subjects reported an angular letter first in some of the trials (although not as frequently as in Experiment 2). These trials were included in the analyses because a preliminary inspection indicated that they produced a pattern of results similar to that of the other trials. Reducing exposure duration was successful in limiting the amount of information extracted from the display. Only 1 subject reported the two curved letters in all trials. For the remaining 9 subjects, only one curved letter was reported on a substantial proportion of trials, ranging from 
Table 3

Experiment 3: Mean Number of Letters per Trial Reported in Each Category in Addition to Target(s)

\begin{tabular}{ccccc}
\hline $\begin{array}{c}\text { No. of } \\
\text { Target Letters } \\
\text { Reported }\end{array}$ & Location & Neutral & & \multicolumn{2}{c}{ Error } \\
\cline { 5 - 6 } One & 1.38 & 1.01 & 0.12 & 0.06 \\
& $0.55^{*}$ & & & \\
Two & 0.64 & 0.38 & 0.11 & 0.21 \\
\hline
\end{tabular}

*Uncorrected mean.

$30 \%$ to $89 \%$, with an overall mean of $57 \%$. Table 3 shows the mean number of correct letters per trial reported in addition to the target(s), as well as the mean number of errors, separately for the reported one-target and twotarget trials. Separate analyses were conducted for the two types of trials. For the one-target trial, the mean number of reported location letters was multiplied by 2.5 for each subject, because there were two possible location letters and five possible neutral letters. The analysis for these trials indicated that location letters were reported more often than neutral letters $[t(8)=1.97, p<.05]$.

For the two-target trials, location letters were defined as those adjacent to any of the curved letters. Some of the displays (those in which the two curved letters were separated by two intervening angular letters) included four possible location letters and three possible neutral letters. The others (those in which the two curved letters were separated by one intervening angular letter) included three possible location letters and four possible neutral letters. These proportions were adjusted individually for each display by multiplying the number of reported location letters by either $4 / 3$ or $3 / 4$. The analysis for these trials indicated that location letters were reported more frequently than neutral letters; but the effect was only marginally significant $[t(9)=1.80, p<.06]$.

These results clearly show a strong tendency for subjects to focus attention on single locations of the display under restrictive conditions that should encourage subjects to attend to the shape of the targets rather than their locations. The results for the one-target trials (in which only one curved letter could be identified) clearly suggest that attending to the location of one curved letter (and reporting letters adjacent to it) was done at the expense of reporting the second curved letter. Therefore, this mode of processing, which evidently conflicted with the demands of the task, appears to represent a structural characteristic of the attentional system, rather than a strategic option.

\section{GENERAL DISCUSSION}

The results clearly indicate that when instructed to report a target specified by color or by shape, subjects attend primarily to its location. Thus, the direction of attention to a relevant spatial location seems to be a general and mandatory process that takes place irrespective of the dimension according to which the stimulus was initially selected for processing.
Our particular method may have grossly underestimated the effect of attending to stimulus location. First, we inferred the effect by measuring residual attention allocated to neighboring locations, rather than to the relevant location itself. Second, even if the subjects initially focused their attention exclusively on the location of the target, they could have subsequently reoriented their attention to neutral letters in the display. Therefore, the proportion of reported location letters relative to neutral letters may well underestimate the extent of focusing attention on target location. Third, various responses classified in the neutral category may in fact belong to the location category. For example, suppose that on a given trial, a subject reports three adjacent letters from left to right. The first letter is the target and the second is recorded as a location response. The third letter would be recorded as a neutral response, since it is removed from the target letter, although it clearly may represent a mode of processing dictated by directing attention on the basis of location. Moreover, informal observations suggested that some of our subjects tended to report letters diametrically opposite to the target. These responses were classified in the neutral category, although they, too, may reflect a process of directing attention on the basis of location or on the basis of figural properties (e.g., symmetry) of the entire display. In spite of all these factors that reduce the proportion of recorded location letters relative to neutral ones, we observed consistently, in all three experiments, highly significant differences between location letters and neutral letters reported. Hence, it is evident that reporting of a target specified by color or by shape is accompanied by a fairly dominant process of attending to the location of the target.

Because, in Experiments 2 and 3, the selection and response criteria were confined to the shape dimension, it is clear that this effect is not limited to tasks requiring the integration of features from different dimensions (e.g., Nissen, 1985), but is involved in the perception of single features as well. This finding is consistent with recent evidence (Prinzmetal, Presti, \& Posner, 1986) indicating that directing attention to stimulus location prior to stimulus onset facilitates the perception of its features and not only the perception of their integration. The present findings strengthen and extend the notion that attention operates as a spotlight, in that they demonstrate that attention is allocated to the vicinity of a relevant stimulus even when it is cued by color or by shape rather than by location. Previous studies have been criticized on the grounds that effects of location preknowledge could be interpreted alternatively as resulting from reduced uncertainty about some dimension of the stimulus (e.g., Davis, Kramer, \& Graham, 1983), that similar facilitatory effects should be obtained with advance knowledge of other stimulus properties, and that even if these effects are not obtained this could be due to greater discriminability of values along the location dimension compared with those of other dimensions (e.g., Duncan, 1984). None of these criticisms can be applied to the present study, in which location was irrelevant to the task and the only prior information given 
to subjects concerned the color or shape of the specified targets.

The present findings are predicted by neither traditional (e.g., Broadbent, 1958) nor modern (e.g., LaBerge, 1975) conceptions of selective attention. It is commonly held that selective processing is accomplished by continuously distinguishing relevant from irrelevant information on the basis of the attribute of selection. For example, when instructed to attend to red items in a multicolor display, one could possibly activate internal structures that respond to the color red by reducing the threshold of these structures or by attenuating structures that respond to different colors.

The alternative view proposed here is that visual space uniquely constitutes a primary "channel" on the basis of which relevant and irrelevant information are kept separate in the course of visual selective processing. An accumulated body of evidence suggests that this channel operates as a spotlight that illuminates an area within which stimuli are processed in detail (e.g., Eriksen \& Hoffman, 1974; Posner, 1980) and outside of which facilitation may gradually decline with greater distance from the attended location (Downing \& Pinker, 1985). The spotlight can assume a small area of a given size (Eriksen \& Hoffman, 1973), or it may increase its size at the cost of a loss of resolution (Eriksen \& St. James, 1986; Eriksen \& Yeh, 1985), or it may flexibly adjust its size to fit the demands of the task (Humphreys, 1981b). However, the operation of this mechanism is strictly controlled by the space dimension in such a way that, for example, even the processing of unrelated stimuli within the illuminated area would be facilitated (e.g., Eriksen \& Hoffman, 1973; Hoffman \& Nelson, 1981). The activation of the spotlight can be controlled by various means. It can be directed by a central cue pointing to a given location in space (e.g., Posner et al., 1980). It can automatically be attracted to the location of a peripheral cue (e.g., Jonides, 1981). It can also, as the present results suggest, be directed to the location of a stimulus that was initially selected on the basis of a different property (e.g., a red target in a multicolor display calls attention to its location). Thus, the enhanced selective processing of items defined by color or by shape is accomplished not by the operation of internal structures representing these selection attributes, but by increasing the sensitivity of the locations the items occupy in space.

Admittedly, the present findings do not provide conclusive support for the above proposition. First, this proposition is based on a single experimental paradigm; further evidence may provide converging operations that would extend the phenomenon beyond task dependence. Second, although the present findings clearly show that attention to target location accompanies selective processing, they do not show that attention to location is a prerequisite for processing of the target. Third, this proposed sequence of processing does not seem to be a particularly parsimonious operation of the attentional system. Given that initial selection must be performed on the basis of distinguishing the relevant color or shape, it is not clear why the activation of internal structures representing these dimensions would not persist for the entire selective act, but should involve another dimension that is irrelevant to the task.

Because of these arguments, the above proposition is presented here as a hypothesis that might be further substantiated, modified, or explained by future research.

\section{REFERENCES}

Barber, P. J., \& Folkard, S. (1972). Reaction time under stimulus uncertainty with response certainty. Journal of Experimental Psychology, 93, 138-142.

BARON, J. (1973). Perceptual dependence: Evidence for an internal threshold. Perception \& Psychophysics, 13, 527-533.

Broadbent, D. E. (1958). Perception and communication. London: Pergamon Press.

Davis, E. T., Kramer, P., \& Graham, N. (1983). Uncertainty about spatial frequency, spatial position, or contrast of visual patterns. Perception \& Psychophysics, 33, 20-28.

Downing, C. J., PINker, S. (1985). The spatial structure of visual attention. In M. I. Posner \& O. S. Marin (Eds.), Attention and performance XI (pp. 171-187). Hillsdale, NJ: Erlbaum.

Duncan, J. (1981). Directing attention in the visual field. Perception \& Psychophysics, 30, 90-93.

DunCAN, J. (1984). Selective attention and the organization of visual information. Joumal of Experimental Psychology: General, 113, 501-517.

ERIKSEN, C. W., \& Hoffman, J. E. (1973). The extent of processing of noise elements during selective encoding from visual displays. $P$ erception \& Psychophysics, 14, 155-160.

ErIKSEN, C. W., HofFMAN, J. E. (1974). Selective attention: Noise suppresion or signal enhancement? Bulletin of the Psychonomic Society, 4, 587-589.

ErIKSEN, C. W., \& St. James, J. D. (1986). Visual attention within and around the field of focal attention. Perception \& Psychophysics, 40, 225-240.

ERIKSEN, C. E., \& YEH, Y. Y. (1985). Allocation of attention in the visual field. Joumal of Experimental Psychology: Human Perception \& Performance, 5, 583-597.

EsTES, W. K. (1972). Interaction of signal and background variables in visual processing. Perception \& Psychophysics, 12, 278-286.

FRYKLUND, J. (1975). Effects of cued-set spatial arrangement and targetbackground similarity in the partial-report paradigm. Perception \& Psychophysics, 17, 375-386.

Hoffman, J. E., \& Nelson, B. (1981). Spatial selectivity in visual search. Perception \& Psychophysics, 30, 283-290.

Hoffman, J. E., Nelson, B., \& Houck, M. R. (1983). The role of attentional resources in automatic detection. Cognitive Psychology, 15, 379-410.

Humphreys, G. W. (1981a). Flexibility of attention between stimulus dimensions. Perception \& Psychophysics, 30, 291-302.

HUMPhREYS, G. W. (1981b). On varying the span of visual attention: Evidence for two modes of spatial attention. Quarterly Journal of Experimental Psychology, 33A, 17-30.

JoNIDES, J. (1981). Voluntary versus automatic control over the mind's eye's movement. In J. B. Long \& A. D. Baddeley (Eds.), Attention and performance IX (pp. 187-204). Hilisdale, NJ: Erlbaum.

Kahneman, D., \& HeNIK, A. (1977). Effects of visual grouping on immediate recall and selective attention. In S. Dornic (Ed.), Attention and performance VI (pp. 307-322). Hillsdale, NJ: Erlbaum.

Kahneman, D., \& HeNiK, A. (1981). Perceptual organization and attention. In M. Kubovy \& J. R. Pomerantz (Eds.), Perceptual organization (pp. 181-211). Hillsdale, NJ: Erlbaum. 
LABERGE, D. (1975). Acquisition of automatic processing in perceptual and associative learning. In P. M. A. Rabbitt \& S. Domic (Eds.), Attention and performance V (pp. 50-64). New York: Academic Press.

LAPPIN, J. S. (1967). Attention in the identification of stimuli in complex displays. Journal of Experimental Psychology, 75, 321-328.

Nissen, M. J. (1985). Accessing features and objects: Is location special? In M. I. Posner \& O. S. Marin (Eds.), Attention and performance XI (pp. 205-219). Hillsdale, NJ: Erlbaum.

POSNER, M. I. (1980). Orienting of attention. Quarterly Journal of Experimental Psychology, 32, 3-25.

Posner, M. I., SNyder, C. R. R., \& Davidson, B. J. (1980). Attention and the detection of signals. Journal of Experimental Psychology: General, 109, 160-174.
Prinzmetal, W., Presti, D. E., \& Posner, M. I. (1986). Does attention affect visual feature integration? Journal of Experimental Psychology: Human Perception \& Performance, 12, 361-370.

SAGI, D., \& JULESZ, B. (1985). "Where" and "what" in vision. Science, 228, $1217-1219$.

SNYDER, C. R. R. (1972). Selection, inspection, and naming in visual search. Journal of Experimental Psychology, 92, 428-431.

Treisman, A., Gelade, G. (1980). A feature-integration theory of attention. Cognitive Psychology, 12, 97-136.

TSAL, Y. (1983). On interpreting the effects of location preknowledge: A critique of Duncan. Perception \& Psychophysics, 34, 297-298.

von WriGHT, J. M. (1970). On selection in visual immediate memory. Acta Psychologica, 33, 280-292.

(Manuscript received August 5, 1987;

revision accepted for publication December 7,1987 .)

\title{
Announcement
}

\author{
29th Annual Meeting of the Psychonomic Society \\ Chicago, Illinois \\ November 10-12, 1988
}

The 29th Annual Meeting of the Psychonomic Society will be held in Chicago, November 10-12, 1988. The meetings will begin Thursday morning and continue until Saturday at noon. The headquarters hotel will be the Palmer House.

The Call for Papers was mailed to members of the Society in April, with a deadline of June 24, 1988, for the receipt of abstracts. The program and hotel reservation cards will be mailed to members in September. A copy of the program will be published in the November issue of the Bulletin of the Psychonomic Society.

For further information, please contact the secretary-treasurer of the Society: Michael E. Rashotte, Department of Psychology, Florida State University, Tallahassee, FL 32306-1051 (Telephone: 904-644-2040; BITNET: Rashot@FSU). 